\title{
Measuring breathlessness during histamine challenge: a simple standardized procedure in asthmatic patients
}

\author{
I.D. Bijl-Hofland*, S.G.M. Cloosterman*, H.Th.M. Folgering**, R.P. Akkermans*, \\ H. van den Hoogen*, C.P. van Schayck*
}

\begin{abstract}
Measuring breathlessness during histamine challenge: a simple standardized procedure in asthmatic patients. I.D. Bijl-Hofland, S.G.M. Cloosterman, H.Th.M. Folgering, R.P. Akkermans, H. van den Hoogen, C.P. van Schayck. C)ERS Journals Ltd 1999.

ABSTRACT: One of the problems in research on symptom perception during histamine challenge has been the difficulty in finding both a valid and practical parameter of the "perceptiveness" for bronchoconstriction in a subject. The purpose of this study was to validate whether the slope in the linear regression model between stimulus and sensation during histamine challenge is an appropriate index for the "perceptiveness" for bronchoconstriction by comparing it with the classical Stevens' law.

One hundred and thirty-four asthmatic patients were included in the study and underwent a bronchial challenge with histamine. The relationship between the change in visual analogue scale (VAS) values and the change in forced expiratory volume in one second (FEV1) as percentage of baseline value was analysed by determining both the exponent $n$ in $\triangle \mathrm{VAS}=k \times(\% \Delta \mathrm{FEV} 1)^{n}$ and the slope $\alpha$ in $\triangle \mathrm{VAS}=k+\alpha(\% \Delta \mathrm{FEV} 1)$. The best-fitting line of both the exponential and the linear regression model were determined by the least-squares method in which the percentage explained variation $\left(\mathbf{R}^{2}\right)$ was compared.

The median value of $\mathrm{R}^{2}$ of the exponential regression line and the linear regression line was 0.76 and 0.83 , respectively, and significantly different. The Spearman rank correlation coefficient between exponent $n$ in the exponential model $\Delta \mathrm{VAS}=\boldsymbol{k} \times(\% \Delta$ FEV1 $)^{n}$ and the slope $\alpha$ in the linear regression model $\Delta V A S=k+\alpha(\% \Delta F E V 1)$ was 0.87 (95\% confidence interval $0.83-0.91)$.

On the basis of the results, it was concluded that the linear regression coefficient can be used as a valid expression to describe the "perceptiveness" of an asthmatic subject instead of Stevens' power function during histamine challenge.
\end{abstract}

Eur Respir J 1999; 13: 955-960.

\author{
*Dept of General Practice and Social \\ Medicine, University of Nijmegen, the \\ Netherlands. **Dept of Pulmonary Dis- \\ eases, Dekkerswald, University of Nij-
} megen, the Netherlands.

Correspondence: I.D. Bijl-Hofland

Dept of General Practice and Social Medicine

CARA research group, code 229

University of Nijmegen

P.O. Box 9101

6500 HB Nijmegen

The Netherlands

Fax: 31243617084

Keywords: Asthma

bronchoconstriction

histamine challenge test

perception

Received: April 231998

Accepted after revision November 111998
Poor perception of bronchoconstriction may be a major underlying cause of fatal or near-fatal asthma attacks $[1,2]$. It is important to identify patients with a poor perception of bronchoconstriction $[3,4]$. In this study, the perception of bronchoconstriction is defined as the subjective quantification of stimulus intensity in relation to the physiological change. One of the problems has been the difficulty of measuring the perception of bronchoconstriction and finding an index that is both a valid and practical parameter of the "perceptiveness" of a subject. It is in the interest of daily diagnostic healthcare to use an index that both corresponds with the actual "perceptiveness" of the patient and can also serve for practical use and interpretation by the practitioner.

STEVENS [5] showed that sensation magnitude is a power function of stimulus intensity in the function $\Psi=k \Phi^{n}$ where $\Psi$ is the subjective qualification of the stimulus, $k$ is a scaling constant, $\Phi$ is the physical change that produce the stimulus, and $n$ is the relative sensitivity. This function describes the relationship between stimulus and

For editorial comments see page 943. sensation over a wide range of stimuli. The power function of Stevens has been used to explore several sensations including breathlessness during added loading tests [6-9], but to the authors' knowledge has not been tested during a histamine or methacholine challenge test.

The bronchial challenge test with histamine or methacholine is the most common diagnostic lung function test to measure the bronchial responsiveness of a patient. In addition to the assessment of bronchial responsiveness, the provocation test has been used to induce airway obstruction as a stimulus to measure a subject's perceptiveness of the associated sensation [10-18]. Some of these studies have used linear regression analysis to describe the relationship between change in forced expiratory volume in one second (FEV1) and visual analogue scale (VAS) values, in which the slope of the regression line $(\alpha)$ was used as the parameter to quantify the "perceptiveness" of the patient [10-13]. The steeper the $\alpha$ the more sensitive a person is to signals of breathlessness. Other studies used the amount of breathlessness score at a 10 or $20 \%$ fall in FEV1 as indicator of the subject's perception [14, 15]. This latter measurement is an index that indicates the 
absolute perceptual magnitude, while the $\alpha$ is used as the parameter that indicates the patient's "sensitivity" towards changes in stimulus.

In the present study, FEV1 was used as an index for airway obstruction and the subjective quantification of breathlessness was measured on a VAS. According to Stevens' law, the relationship between the reduction in FEV1 and VAS value can be described in the power function $\Delta \mathrm{VAS}=k \times(\% \Delta \mathrm{FEV} 1)^{n}$, where $n$ is the relative sensitivity to changes in FEV1. When $n=1$, changes in FEV1 correspond linearly to changes in VAS; when $n>1$, small ranges of FEV1 correspond with a wider (exponential) range of VAS; and when $n<1$, wide ranges of FEV1 correspond with a smaller (exponential) change in VAS. Although the exponent $(n)$ as an expression for the "perceptiveness" of a subject is probably the most valid one available, it is a less practical parameter to use and interpret during a routine histamine challenge test than a linear regression coefficient (slope). Therefore, the "perceptiveness" for bronchoconstriction during a histamine challenge test was studied in asthmatic patients using the linear regression coefficient (slope) between the stimulus and sensation, and this was compared with Stevens' law. The appropriateness of describing the relationship between two variables by a regression analysis (exponential or linear) was analysed by determining the best fit of the particular model. A congruence between the best fit of the linear regression model and the best fit of the exponential model would indicate that the linear regression model can be used to describe the relationship between the stimulus and sensation. This would imply that the identification of poor perceivers can be carried out in a standardized procedure during a routine diagnostic test, by determining the linear regression coefficient.

\section{Methods}

\section{Patient selection}

Patients were recruited from a research project on symptom perception in house dust mite allergic asthmatic patients aged 16-60 yrs, during bronchodilator treatment [19]. Inclusion criteria for this research project were: FEV $1>50 \%$ predicted value; provocative concentration causing a $20 \%$ fall in FEV1 (PC20) on histamine $\leq 8$ $\mathrm{mg} \cdot \mathrm{mL}^{-1}$ and/or reversibility of obstruction $\geq 15 \%$ after inhalation of $800 \mu \mathrm{g}$ salbutamol (compared to baseline FEV1) and patients had to have lower airway symptoms. The present study was performed at the first assessment of lung function parameters after inclusion in the larger trial [19]. Only patients with asthma, with a baseline FEV $1>50 \%$ pred value who qualified for a histamine challenge test, were recruited for the present study. Patients had to have a bronchial provocation test with a minimum reduction in FEV $1 \geq 20 \%$ of baseline value with at least two doubling doses of histamine, to determine a patient's "perceptiveness" during the test. Informed consent was obtained from each patient.

\section{Bronchial provocation test}

Each patient underwent a histamine inhalation test according to European Respiratory Society standards [20].
Prior to testing, no short-acting bronchodilators were used for at least $8 \mathrm{~h}$ and no long-acting bronchodilators for at least $12 \mathrm{~h}$. Doubling concentrations of histamine from 0.03 to $16 \mathrm{mg} \cdot \mathrm{mL}^{-1}$ were administered until $\mathrm{PC} 20$ was achieved. The bronchial response to each dose of histamine was expressed as the reduction in FEV1 as percentage of baseline value.

\section{Assessment of breathlessness}

Breathlessness was measured before each measurement of FEV1 using a VAS. The subjects rated the magnitude of their respiratory sensation on a $100-\mathrm{mm}$ horizontal VAS with the words "minimum" and "maximum" on the left and right side, respectively. The subjects were instructed that the word minimum refers to "no complaints of respiratory sensation such as shortness of breath, breathlessness or chest tightness" and maximum stands for "the worst respiratory complaints imaginable". The subjects were instructed to place a vertical mark on the line, such that its position, relative to the two extremes, indicated the magnitude of respiratory sensation at the moment of the measurement. Every subject was told that the purpose was to measure the magnitude of their breathlessness during the test, and also that the magnitude of "no breathlessness at all" was possible. Previous VAS values were not shown to the patients. VAS values at any dose of histamine were expressed as the difference ( $\mathrm{mm}$ ) compared to baseline [21].

\section{Expressions for the perception of bronchoconstriction}

The relationship between the changes in VAS values and the changes in FEV1 as percentage of baseline value was analysed in two ways. The first index $(n)$ is the exponent in the power function $\Psi=k \Phi^{n}$, where $\Psi$ is the change in VAS value, $\Phi$ is the change in FEV1 as percentage of baseline value, and $k$ is a constant. The slope of the best-fit line between changes in VAS value and changes in FEV1 as percentage of baseline value as a function of $\log \Psi=$ $\log k+n \times \log \Phi$ was taken as the measured exponent $(n)$ in the power function $\Psi=k \Phi^{n}$. To perform the log-transformation, a constant value was added to each value of $\Psi$ (changes in VAS) and $\Phi$ (changes in FEV1). This standard summation was carried out to avoid negative or zero values which have been lost in the analysis. The second index $(\alpha)$ is the slope of the linear regression analysis between changes in VAS values and change in FEV1 as percentage of baseline value. A minimum of three points on the doseresponse curve (in addition to the baseline value) were required in order to perform both regression analyses (exponential and linear).

\section{Analysis}

Comparison of both models. The best-fit lines of both the exponential and the linear regression model were determined by the least-squares method and compared by the percentage explained variation $\left(\mathrm{r}^{2}\right)$. Wilcoxon signed rank tests were performed to compare the best fit of both models. 
Comparison of the sensitivity indices: exponent versus slope. The median value and $25-75$ th percentile range of the perceptiveness score by means of the exponent $n$ and the linear regression slope $\alpha$ are presented. A Spearman rank correlation coefficient was calculated between values of slope $\alpha$ and exponent $n$ in order to investigate whether the most "sensitive" perceivers of bronchoconstriction who, according to the Stevens' power function, are also the most "sensitive" subjects as indicated in the linear function. Calculations of mathematical expressions of the exponent $n$ and the linear regression slope $\alpha$, are obviously more accurate when there are certain mathematical test conditions present. These mathematical conditions and the results, taking into account these test criteria, are described in the Appendix.

Index of the absolute perceptual magnitude. The median value of the perception score at a $20 \%$ fall in FEV1 was taken as the index of absolute perceptual magnitude. The perception score at a $20 \%$ fall in FEV1 with regard to baseline (PS20) was obtained from the VAS response curve by linear interpolation. The relationship between the "sensitivity" index (slope $\alpha$ ) with the "absolute magnitude" index (PS20) was observed by a scatter plot of slope $\alpha$ versus PS20.

\section{Results}

\section{Patients}

One hundred and thirty-four out of a potential 162 asthmatic patients were included. The clinical characteristics of the 134 asthmatic patients who could be analysed are presented in table 1 . Of the 162 subjects in the original trial, five patients had a baseline FEV $1<50 \%$ pred, one subject did not speak Dutch, no data of respiratory sensation were recorded for four patients, nine patients had a provocation test which consisted of only one doubling dose of histamine, and nine patients had a bronchial provocation test with a reduction in FEV1 $<20 \%$ of baseline value.

\section{Perception of respiratory sensation}

There was considerable variability between subjects in the subjective quantification of the stimulus at any level of FEV1 reduction as a percentage of baseline value (fig. 1). The median value of the VAS value at the beginning of the histamine challenge test was $8 \mathrm{~mm}(25-75$ th percentile, $2-18 \mathrm{~mm})$. The median change in VAS value was $27 \mathrm{~mm}(25-75$ th percentile, $16-39 \mathrm{~mm})$ and the median value of the reduction in FEV1 as percentage of baseline value was $26 \%$ (25-75th percentile, $23-31 \%)$. The median maximum VAS value was $41 \mathrm{~mm}$ (25-75th percentile, $26-58 \mathrm{~mm}$ ).

\section{Comparison of the two regression models}

The median value of the $r^{2}$ of the exponential lines was 0.76 (25-75th percentile, $0.58-0.88$ ), and the median value of the $\mathrm{r}^{2}$ of the linear regression lines was $0.83(25-75 \mathrm{th}$
Table 1. - Clinical characteristics of the study population

\begin{tabular}{|c|c|}
\hline & Subjects $(n=134)$ \\
\hline Age* yrs & $34(11)$ \\
\hline Sex F/M & $61 / 73$ \\
\hline $\mathrm{PC}_{20}{ }^{+} \mathrm{mg} \cdot \mathrm{mL}^{-1}$ & $1.18(0.06-16.00)$ \\
\hline $\mathrm{FEV}_{1} * \mathrm{~mL}$ & $3229(724)$ \\
\hline $\mathrm{FEV}_{1} * \%$ pred & $86(15)$ \\
\hline \\
\hline$\beta_{2}$-Agonist (<once a day) & 5 \\
\hline \multicolumn{2}{|l|}{$\begin{array}{l}\text { Combination of } \beta_{2} \text {-agonist and } \\
\text { anticholinergics (Berodual } \AA \text { ) }\end{array}$} \\
\hline$<$ Once a day & 76 \\
\hline Regular ( $\geq$ once a day) & 15 \\
\hline Regular antihistamines (once a day) & 7 \\
\hline \multicolumn{2}{|l|}{ Beclomethasone nose spray } \\
\hline$<$ Once a day & 1 \\
\hline Regular & 1 \\
\hline
\end{tabular}

Data are presesnted as: ${ }^{*}$ : mean $(\mathrm{SD}){ }^{+}$: geometric mean (range). F: female; M: male; FEV1: forced expiratory volume in one second; PC20: provocative concentration causing a $20 \%$ fall in FEV1.

percentile, 0.67-0.91). A Wilcoxon sign rank test between the $r^{2}$ of both models showed a difference in the $r^{2}$ values, in which the $r^{2}$ of the linear model was greater than the $r^{2}$ of the exponential model in 106 cases. In only 27 cases, the $r^{2}$ of the linear model was less than $r^{2}$ of the exponential model $(p=0.0001)$. In one case, the $r^{2}$ of both models were equal. Excluding patients who did not comply with extra statistical conditions did not lead to important different results in the comparison of the two regression models (see Appendix).

"Sensitivity" indices of the perception of changes: slope and exponent

A histogram of the values of the exponent $(n)$ in the power function $\Psi=k \Phi^{n}$ is shown in figure 2. The median value for exponent $(n)$ was $0.85(25-75$ th percentile, $0.51-1.27)$. In 77 (58\%) patients the $n$ was $<1$. These patients scored small changes on the VAS compared with

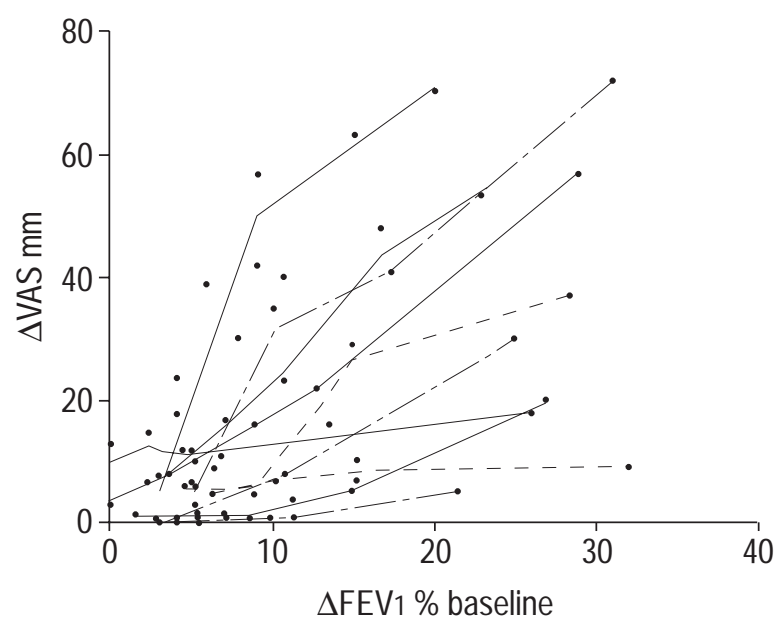

Fig. 1. - Scatter plot of the changes in visual analogue scale (VAS) value and the changes in forced expiratory volume in one second (FEV1) as percentage of baseline value of 10 randomly selected subjects. 


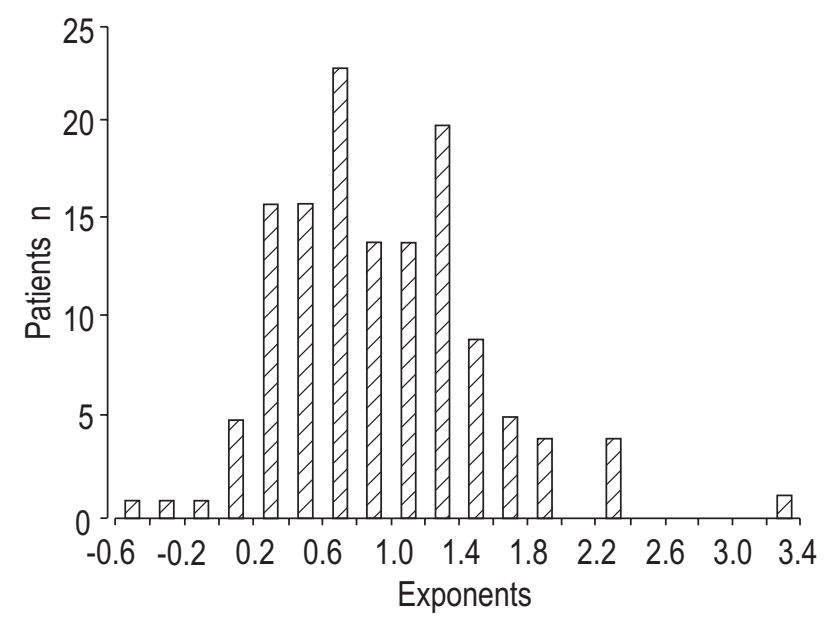

Fig. 2. - Histogram of the values of exponents $n$ as index of the perception of bronchoconstriction during the histamine challenge test. The exponents are assessed in the power function $\Psi=k \Phi^{n}$, where $\Psi$ is the change in visual analogue scale values and $\Phi$ is the change in forced expiratory volume in one second (FEV1) as percentage of baseline value $(\mathrm{n}=134)$. Median exponent $(25-75$ th percentile $)=0.85(0.51-1.27)$.

relatively large changes in the reduction in FEV1 as percentage of baseline value. The other 57 (42\%) patients had $n>1$ and scored relatively large changes on the VAS in spite of small changes in reduction in FEV1 as percentage of baseline value. Two examples of exponential lines are displayed in figure 3. Subject No. 34 was a patient with a relatively high perception of the reduction in FEV1 and subject No. 13 was a patient who was less sensitive to the reduction in FEV1. A histogram of the values of the slopes in the linear regression analysis of the changes in FEV1 as percentage of baseline value and changes in VAS value is presented in figure 4. The median slope was 0.91 (25-75th percentile, 0.48-1.45).

The Spearman rank correlation coefficient between exponent $n$ in the exponential model $\Delta \mathrm{VAS}=k \times(\% \Delta \mathrm{FEV} 1)^{n}$ and the slope $\alpha$ in the linear regression model $\triangle \mathrm{VAS}=$

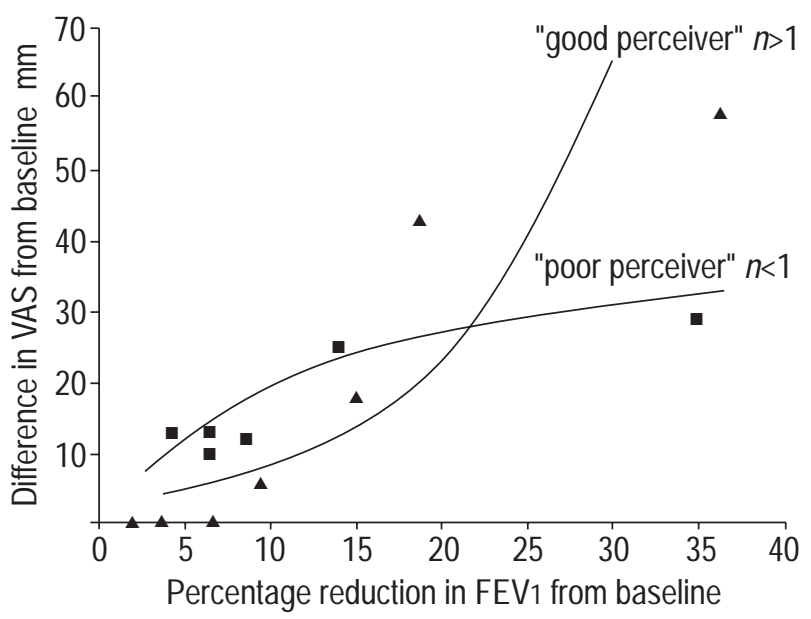

Fig. 3. - Two examples from sensations during a histamine provocation test in two different subjects. The exponents $n$ as index of the perception of bronchoconstriction during the histamine challenge test are assessed in the power function $\Psi=k \Phi^{n}$, where $\Psi$ is the change in visual analogue scale (VAS) value and $\Phi$ is the change in forced expiratory volume in one second (FEV1) as percentage of baseline value. $\boldsymbol{\Delta}$ : subject 34 $(\mathrm{n}=1.62)$; $\mathbf{\square}$ : subject $13(n=0.59)$.

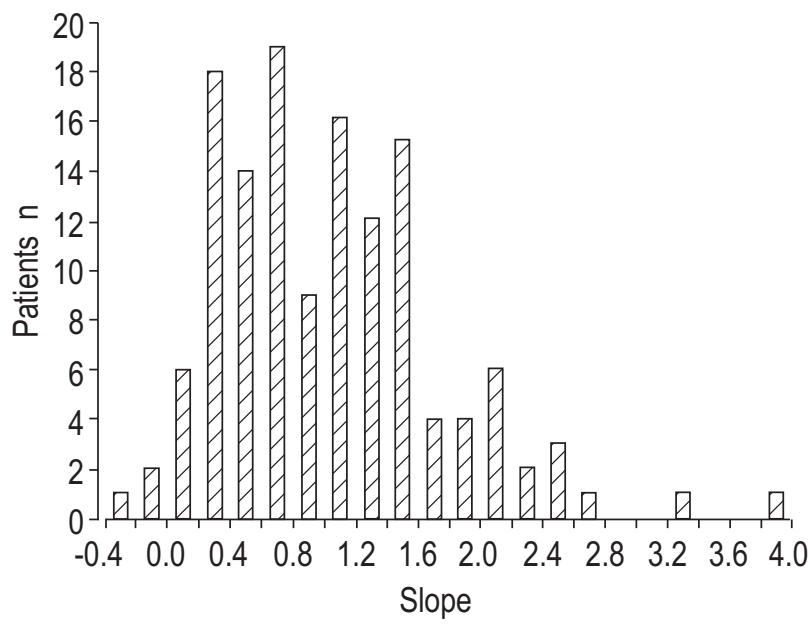

Fig. 4. - Histogram of the values of linear regression slopes $\alpha$ as index of the perception of bronchoconstriction during the histamine challenge test. The slopes are assessed in the linear regression analysis between the change in visual analogue scale value and the change in forced expiratory volume in one second (FEV1) as percentage of baseline value $(n=134)$. Median slope $(25-75$ th percentile $)=0.91(0.48-1.45)$.

$k+\alpha(\% \Delta \mathrm{FEV} 1)$ was 0.87 (95\% CI $0.83-0.91)$. The most "sensitive" perceivers of bronchoconstriction according to Stevens' power function are also the most "sensitive" subjects according to the linear function.

\section{Index of the absolute perceptual magnitude}

The median value of PS20, as the index of the absolute perceptual magnitude, was $35 \mathrm{~mm}$ (25-75th percentile, 21-52 $\mathrm{mm})$. The relationship between the "sensitivity" index (slope $\alpha$ ) with the PS20 is presented in figure 5.

\section{Discussion}

This study was performed to investigate the validity of the linear regression slope $(\alpha)$ in describing the relationship between the stimulus and the subjective quantification

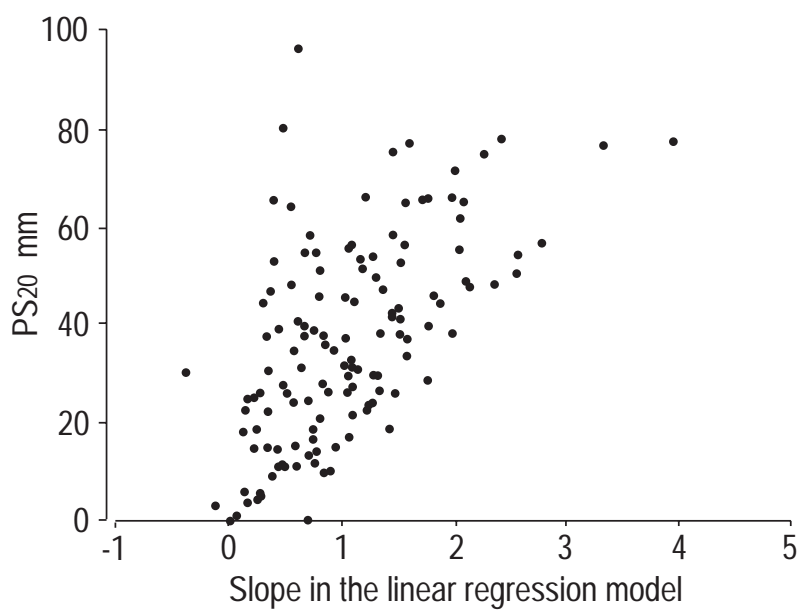

Fig. 5. - Scatter plot of the relationship between the "sensitivity" index (slope $\alpha$ in the linear regression model $\Delta \mathrm{VAS}=k+\alpha(\% \Delta \mathrm{FEV} 1)$ with the "absolute perceptual magnitude" index (perception (VAS) score at a $20 \%$ fall in FEV1; PS20). VAS: visual analogue scale; FEV1: forced expiratory volume in one second. 
of the associated sensation during a histamine challenge test by comparing it with the relationship expressed by the classical power function of Stevens which is theoretically the most appropriate description. The best fit of both the exponential line and the linear regression line were compared by determining the percentage explained variation in the response variable of each model. The median values of $r^{2}$ of the exponential regression line and the linear regression line were 0.76 and 0.83 , respectively, and differed significantly. This result indicates that the linear regression line described more variation in the subjective quantification of the sensation than the exponential function of Stevens. Although the exponent in Stevens' power function is assumed to describe the relationship between stimulus and sensation in the most appropriate way, the linear regression coefficient (slope) appears to be a better alternative in the specific situation of a bronchial challenge test in this group of asthmatic patients. A possible cause of this difference in the percentage explained variation might be the small stimulus range of a bronchial challenge test in which the Stevens' power function approach was applied. The application of the Stevens' power function requires the entire stimulus range. The bronchial challenge test in clinical practice is only applied in the first part of the stimulus range (histamine administration was stopped after the FEV1 had fallen $\geq 20 \%$ ). The high percentage explained variation in the response variable by the linear regression model when compared with the exponential model is apparently caused by the fact that the first part of the total response curve to the stimulus can be described by a linear relationship. The linear regression coefficient may be a less accurate indicator of the perceptiveness for bronchoconstriction when the reduction in FEV1 is $>20 \%$. Thus, it appears that during the histamine challenge test, the linear regression coefficient provides both a practical and valid index to describe the relationship between stimulus and sensation. The regression coefficient can easily be translated for a clinician into a practical interpretation of this "perceptiveness" index.

The study of Boulet et al. [14] used the PS20 as a calculated measurement of the perception of bronchial challenge. This measurement indicates a patient's absolute perceptual magnitude and does not include the "sensitivity" towards changes in stimulus. On the other hand, the slope as a sensitivity index gives no indication of absolute perceptual magnitude. There may be patients with a high sensitivity in combination with a low absolute perceptual magnitude. These patients perceive the changes in stimulus but estimate the respiratory distress as being less of an inconvenience than patients with a high absolute perceptual magnitude. It is also possible that there are patients with a low sensitivity and a high absolute perceptual magnitude. These patients estimate their respiratory distress as very inconvenient but can poorly differentiate between different degrees of respiratory distress. Therefore, both indices give additional information about the symptom perception of a patient.

The determination of the perceptiveness for bronchoconstriction during a histamine challenge test can only be performed in asthmatic patients with a FEV $1>50 \%$ pred and with a positive histamine challenge test with at least two doubling doses of histamine. Consequently, there will still be a group of subjects in which the perceptiveness for bronchoconstriction cannot be determined during the his- tamine challenge test with a regression model. In patients with a baseline $\mathrm{FEV} 1<50 \%$ pred and very hyperresponsive patients who need only one or two doses of inhaled histamine to induce a large reduction in FEV1 (and the opposite, in subjects who do not respond to histamine), the perceptiveness for bronchoconstriction cannot be determined from a histamine challenge test. This is a clear limitation of this method of using the challenge test as patients with a more severe degree of asthma are especially of interest in determining their perception of symptoms because they are the group most at risk.

RoISMAN et al. [11] observed that corticosteroid treatment was associated with improved perception of bronchoconstriction. However, none of the subjects in the present study used inhaled corticosteroids. Therefore, the present results about the perception of bronchoconstriction might be different from the perception of a population of asthmatic subjects using corticosteroids.

Breathlessness is not only a result of FEV1 as a derivative of airway resistance but is also influenced by many other factors such as lung volume, respiratory muscle strength and respiratory muscle effort [22]. Moreover, histamine (which was used as a stimulus to induce bronchoconstriction and generate respiratory sensation) also stimulates various other receptors, Furthermore, a person's perceptiveness also reflects their attitudes, expectations and tolerance [23].

The present results show that, although the exponent $n$ in Stevens' power function $\Psi=k \Phi^{n}$ is the classical description of the relationship between stimulus and sensation, the linear regression coefficient (slope $\alpha$ ) provides daily diagnostic healthcare with a simple alternative index for the perception of bronchoconstriction during a histamine challenge test. The relatively high percentage of explained variation in the subjective sensation quantification by the linear model compared with that in the exponential model advocates the use of the slope $(\alpha)$ instead of the exponent $(n)$. It provides the clinician with a practical index for interpreting a patient's "perceptiveness" for bronchoconstriction during a routine histamine challenge test.

\section{Appendix}

Calculations of mathematical expressions of the exponent $n$ and the linear regression slope $\alpha$ are more accurate when more observations are available and when the correlation coefficient between the values of VAS and FEV1 is $>0.71$. The latter criterion corresponds to a situation in which $\geq 50 \%$ of the total variation in changes in VAS value are explained by the changes in FEV1 [11]. Excluding data which do not meet these criteria might introduce a recruitment bias. Patients with a correlation coefficient $<0.71$ between the values of VAS and FEV1 might be the poor perceivers, and those with a provocation test with few doubling doses of histamine are the most hyperresponsive. In order to assess the external validity of the results, the analysis was performed with and without data which did not comply with one or two of the following conditions: 1 ) a correlation coefficient of $>0.71$ between the changes in VAS values and the changes in FEV1 as percentage of baseline value; and 2) a bronchial provocation test with at least three doubling dose of histamine. The findings of this 
analysis were compared with the results of the total study population.

In six patients, the bronchial provocation test consisted of only two doubling doses of histamine. In 27 patients, the correlation coefficient of the linear regression between the $\log$ values of changes in VAS values and the log values of changes in FEV1 as percentage of baseline value was $<0.71$. In 16 patients, the correlation coefficient of the linear regression between the change in VAS values and the reduction in FEV1 as percentage of baseline value was $<0.71$. The analysis with the exponent $n$ was finally carried out in both 134 and 101 patients and with the linear regression slope $\alpha$ in 134 and 112 patients. The latter groups of 101 and 112 patients complied with the extra statistical conditions for calculating, respectively, the exponent $n$ and the linear regression slope $\alpha$.

The reduction of the size of the study population by excluding patients who did not comply with these extra statistical conditions did not lead to important different results in the comparison of the two regression models. The median value of $\mathrm{r}^{2}$ of the exponential lines was 0.81 (25-75th percentile, $0.70-0.89)$, and the median value of $\mathrm{r}^{2}$ of the linear regression lines was 0.84 (25-75th percentile, 0.73-0.91). A Wilcoxon sign rank test between the $r^{2}$ values of both models showed a difference in $r^{2}$ values, in which the $r^{2}$ of the linear model was greater than $r^{2}$ of the exponential model in 79 cases. In only 20 cases was the $r^{2}$ of the linear model less than the $r^{2}$ of the exponential model $(\mathrm{p}=0.0001)$.

The median value for exponent $n$ in the power function $\Delta \mathrm{VAS}=k \times(\% \Delta \mathrm{FEV} 1)^{n}$ was $0.94(25-75$ th percentile, 0.66-1.29) in the group of 101 patients who complied with the extra statistical conditions for calculating the exponent. The median value was 0.85 (25-75th percentile, $0.25-1.27)$ for the total study population. The median value for slope $\alpha$ in the linear regression function $\triangle \mathrm{VAS}=$ $k+\alpha(\% \Delta \mathrm{FEV} 1)$ was 1.05 (25-75th percentile, $0.59-1.49)$ in the group of 112 patients which complied with the extra statistical conditions for calculating the slope. The median value was 0.91 (range $0.48-1.45$ ) for the total study population. The Spearman rank correlation coefficient between exponent $n$ in the exponential model and the slope $\alpha$ in the linear regression model remains 0.87 (95\% CI 0.81-0.91), the same as for the total study population.

\section{References}

1. Barnes PJ. Poorly perceived asthma. Thorax 1992; 47: 408-409.

2. Kikuchi Y, Okabe S, Tamura G, et al. Chemosensitivity and perception of dyspnea in patients with a history of near-fatal asthma. N Engl J Med 1994; 330: 1329-1334.

3. Barnes PJ. Blunted perception and death from asthma. $N$ Engl J Med 1994; 330: 1383-1384.

4. Rubinfeld AR, Pain MCF. Perception of asthma. Lancet 1976; 24: 882-884.

5. Stevens SS. Psychophysics: Introduction to its Perceptual, Neural, and Social Prospects. New York, John Wiley, 1975; pp. 1-329.

6. Killian KJ, Bucens DD, Campbell EJM. Effect of breathing patterns on the perceived magnitude of added loads to breathing. $J$ Appl Physiol 1982; 52: 578-584.

7. Gottfried SB, Altose MD, Kelsen SG, Cherniack NS.
Perception of changes in airflow resistance in obstructive pulmonary disorders. Am Rev Respir Dis 1981; 124: 566570.

8. Tack M, Altose MD, Cherniack NS. Effect of aging on respiratory sensations produced by elastic loads. $J$ Appl Physiol 1981; 50: 844-850.

9. Mahler DA, Harver A, Rosiello R, Daubenspeck JA. Measurement of respiratory sensation in interstitial lung disease. Chest 1989; 96: 767-771.

10. Brand PLP, Rijcken B, Schouten JP, Koëter GH, Weiss ST, Postma DS. Perception of airway obstruction in a random population sample. Relationship to airway hyperresponsiveness in the absence of respiratory symptoms. Am Rev Respir Dis 1992; 146: 396-401.

11. Roisman GL, Peiffer C, Lacronique JG, Le Cae A, Dusser DJ. Perception of bronchial obstruction in asthmatic patients. Relationship with bronchial eosinophilic inflammation and epithelial damage and effect of corticosteroid treatment. J Clin Invest 1995; 96: 12-21.

12. Marks GB, Yates DH, Sist M, et al. Respiratory sensation during bronchial challenge testing with methacholine, sodium metabisulphite, and adenosine monophosphate. Thorax 1996; 51: 793-798.

13. Burdon JGW, Juniper EF, Killian KJ, Hargreave FE, Campbell EJM. The perception of breathlessness in asthma. Am Rev Respir Dis 1982; 126: 825-828.

14. Boulet LP, Cournoyer I, Deschesnes F, Leblanc P, Nouwen A. Perception of airflow obstruction and associated breathlessness in normal and asthmatic subjects: correlation with anxiety and bronchodilator needs. Thorax 1994; 49: 965-970.

15. Turcotte H, Corbeil F, Boulet LP. Perception of breathlessness during bronchoconstriction induced by antigen, exercise, and histamine challenges. Thorax 1990; 45: 914-918.

16. Connolly MJ, Crowley JJ, Charan NB, Nielson CP, Vestal RE. Reduced subjective awareness of bronchoconstriction provoked by methacholine in elderly asthmatic and normal subjects as measured on a simple awareness scale. Thorax 1992; 47: 410-413.

17. Boner AL, De Stefano G, Piacentini GL, et al. Perception of bronchoconstriction in chronic asthma. J Asthma 1992; 29: $323-330$.

18. Lougheed MD, Lam M, Forkert L, Webb KA, O'Donnell DE. Breathlessness during acute bronchoconstriction in asthma. Am Rev Respir Dis 1993; 148: 1452-1459.

19. van Schayck CP, Cloosterman SGM, Hofland ID, van Herwaarden CLA, van Weel C. How detrimental is chronic use of bronchodilators in asthma and chronic obstructive pulmonary disease. Am J Respir Crit Care Med 1995; 151: 1317-1319.

20. Sterk PJ, Fabbi LM, Quanjer $\mathrm{PhH}$, et al. Airway responsiveness: standardized challenge testing with pharmacological, physical and sensitizing stimuli in adults. Eur Respir J 1993; 6: 53-83.

21. Peiffer C, Toumi M, Razzouk H, Marsac J, Lockhart A. Relationship between spontaneous dyspnoea and lability of airway obstruction in asthma. Clin Sci 1992; 82: 71772.

22. Killian KJ, Campbell EJM. Mechanisms of dyspnea. In: Mahler DA, ed. Dyspnea. New York, Futura, 1990; pp. 55-73.

23. Redelmeier DA, Rozin P, Kahneman D. Understanding patients' decisions: cognitive and emotional perspectives. J Am Med Assoc 1993; 270: 72-76. 